\title{
Postcoalescence Evolution of Growth Stress in Polycrystalline Films
}

\author{
A. González-González, ${ }^{1, *}$ C. Polop, ${ }^{2}$ and E. Vasco ${ }^{1}$ \\ ${ }^{1}$ Instituto de Ciencia de Materiales de Madrid, Consejo Superior de Investigaciones Científicas (CSIC), 28049 Madrid, Spain \\ ${ }^{2}$ Departamento de Física de la Materia Condensada, Universidad Autónoma de Madrid, 28049 Madrid, Spain
}

(Received 27 July 2012; revised manuscript received 3 December 2012; published 31 January 2013)

\begin{abstract}
The growth stress generated once grains coalesce in Volmer-Weber-type thin films is investigated by time-multiscale simulations comprising complementary modules of (i) finite-element modeling to address the interactions between grains happening at atomic vibration time scales $(\sim 0.1 \mathrm{ps})$, (ii) dynamic scaling to account for the surface stress relaxation via morphology changes at surface diffusion time scales $(\sim \mu \mathrm{s}-\mathrm{ms})$, and (iii) the mesoscopic rate equation approach to simulate the bulk stress relaxation at deposition time scales $(\sim \sec -\mathrm{h})$. On the basis of addressing the main experimental evidence reported so far on the topic dealt with, the simulation results provide key findings concerning the interplay between anisotropic grain interactions at complementary space scales, deposition conditions (such as flux and mobility), and mechanisms of stress accommodation-relaxation, which underlies the origin, nature and spatial distribution, and the flux dependence of the postcoalescence growth stress.
\end{abstract}

DOI: 10.1103/PhysRevLett.110.056101

PACS numbers: 68.55.J-, 68.60.Bs, 81.15.Aa

The uncontrolled internal field of growth stress generated once the grains have coalesced upon the film closure stage represents a major endemic flaw on the in-service reliability of thin-film-based integrated devices, multilayer systems, and coatings to operate in conditions of high temperatures and overpressure [1-4]. Under these conditions the growth stress is magnified to reach greater values than those of the film cohesion and interfacial adhesion forces, which induce phenomena of film fracture, delamination, mechanical fatigue, and peeling off [1-3]. In this circumstance, the understanding of the nature and behavior of the postcoalescence growth stress-with the goal of tailoring it-represents a major technological challenge.

While there is wide consensus on the origin of the precoalescence growth stresses as a result of the balance between surface tension and film cohesion forces (the former prevails for early growth stages in which the Laplace pressure compresses to the isolated grains [5], while the latter accounts for the traction forces between close grains for later stages [6]), the nature of the postcoalescence growth stress is still a matter of discussion [5-12]. This debate arises owing to the difficulty of finding a model that consistently addresses the following main experimental evidences for systems growing in high mobility conditions: (I) The postcoalescence stress is compressive and (II) flux dependent, this dependence being reversible $[5-8,10]$. It means that the compressive stress relaxes asymptotically, or even becomes tensile if the deposition flux is stopped, and it is regenerated once resumed. (III) The postcoalescence morphology (imaged by high-resolution microscopy able to discern between different types of intergrain regions) is formed by irregular multigrain structures-identified as grain bundles in which shallow internal grain boundaries (GBs) and noncoherent crystalline facets are visible - separated from each other by deep grooves and discontinuities. This morphology (baptized in Ref. [13] as "inside bundling-outside grooving") that does not correspond to the typical percolative morphology resulting from the random grain coalescence; it has been reported for evaporated polycrystalline films of Fe [5], $\mathrm{Au}$ [13], $\mathrm{Cu}$ [14], Ag [15], Al [16], and Sn [17]. (IV) The formation of such structures involves the generation (irreversible at short-range and reversible at long one) of in-plane texture via grain reorientation as revealed by surface-orientation maps and $\phi$-scan x-ray diffraction (XRD) [13]. Such a reorientation happens via generation of shear strain in larger grains than the critical size $(\sim 100 \mathrm{~nm})$ for hindering grain rotation.

Another ingredient to consider is the key role of the film surface on the stress behavior for the studied systems (namely, polycrystalline films growing in the VolmerWeber mode). Indeed, the previous models explain the generation of the compressive postcoalescence stress as a consequence of: [(a) Koch et al. [5]] the Laplace pressure caused by the surface tension that is recovered once the cohesion forces drop as the grown film becomes continuous, such that both the early-growth stage and the postcoalescence stress would have a common origin, [(b) Tello et al. and Shin et al. [6,7]] the reversible insertion of adatoms into the GBs via surface currents biased by the surface curvature and stress gradients, and [(c) Friesen et al. and Lieb et al. [8,9]] the elastic strain field that experiences a surface in the presence of an impinging flux due to changes in the adatom population. Beyond the virtues and defects of these models (see discussion in Ref. [9]), all of them fail to satisfactorily explain the evidences (III) and (IV). In this context, a recent model (González-González et al. [13]) based on feature sizeselective interactions between grains and/or structures was proposed to address the inside bundling-outside 
grooving morphology in annealed Au films grown ad hoc to minimize the substrate mechanical constrictions. This model suggests that the behavior of postcoalescence growth stress is the result of the balance between mechanisms of relaxation (via surface currents) and stress accommodation (by generation of both radial and shear strain) at different space scales.

In this Letter, we investigate the implications of the "inside bundling-outside grooving" model in regard to elucidate the origin, nature and spatial distribution, evolution, and flux dependence of the postcoalescence growth stress by time-multiscale simulation, since the involved processes (namely, strain generation, surface currents, GB diffusion, and deposition) take place at largely different time scales.

Model based on evidence (IV). - The morphology of the polycrystalline film is idealized as formed by a compact array of rounded elastic grains, which are separated from each other by GBs that emerge toward the surface at different heights, generating a network of narrow gaps between grains (with the gap width $\Delta \ll$ grain size $\lambda$ ). The model assumes that such grains interact with each other as follows: the sections within the gaps between grains are under traction generated by the cohesion forces (long-range interactions along the radial direction $\hat{R}$ ), whereas the buried sections (just below the grain junction) are under shear loads (torques) generated by the in-plane misorientation between grains (short-range interactions along the azimuthal direction $\hat{\phi}$ ). As a consequence of these interactions, a growth stress $\sigma$ arises whose component normal to the surface (along the unit vector $\hat{N}$ ) is $\sigma_{N} \approx-\left(\hat{\nabla}_{S} \cdot \gamma\right) \hat{N}$ with $\hat{\nabla}_{s}$ denoting the surface gradient vector and $\gamma$ being the resulting force from the balance between the involved surface-tension forces [Fig. 1(a)] along the out-of-the-film plane direction $(\hat{z})$,

$$
\gamma=2 \gamma_{s} \sin [\arctan (m)]-\gamma_{g b}(\theta)-\gamma_{e}(\Delta, \theta, \lambda),
$$

where $\gamma_{s}$ represents the surface energy with $\arctan (m)$ being the wetting angle at the grain junction; $\gamma_{g b}$ corresponds to the GB energy depending on the misorientation angle $\theta$ between neighboring grains; and $\gamma_{e}$ is the accumulated elastic energy during the coalescence to what both the radial strain (that is $\propto \Delta / \lambda$, the width of the gap to close) and shear one ( $\propto \theta$, the angle to twist) contribute. Beyond a detailed description of the $(\Delta, \theta, \lambda)$ dependence of $\gamma_{e}$ provided elsewhere $[13,18]$, the key points to bear in mind for the model proposed in this Letter are: (a) the shear-strain contribution to $\gamma_{e}$ rises as the coalescing grains are larger since their capacity to rotate drop as $\propto 1 / \lambda^{2}$ [19], and (b) since the mechanical coupling of two grains is better as the GB in between emerges at a higher height (i.e., at a shallower gap), the model assumes that the zipping of a given GB must be accompanied by the generation of shear strain within the whole crystalline volume that extends beyond the neighboring grains across
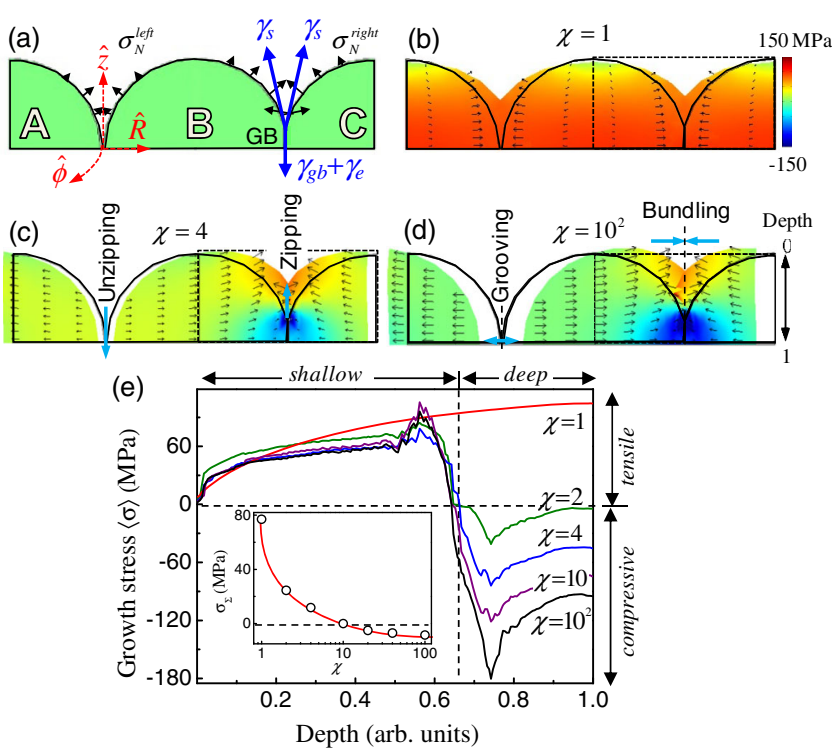

FIG. 1 (color online). (b)-(d) Elastic deformation profiles of three nonevenly spaced grains computed by FEM from initial profile in (a) for different $\chi$. The arrows and the color scale in (b)-(d) depict the local fields of displacements and growth-stress strength, respectively; whereas the solid curves correspond to the initial profile. (e) Depth profiles of the longitudinal-averaged growth stress $\langle\sigma\rangle$ fields within the enclosed regions in (b)-(d) (i.e., around $\mathrm{GB}+$ gap between $B-C$ ) for $\chi$ ranging between 1-100. Inset: $\chi$ dependence of $\sigma_{\Sigma}$ estimated as $\sigma_{\Sigma} \propto \int_{\text {depth }}\langle\sigma\rangle$, areas under the plots in (e). Note that the profiles in (a)-(d) do not keep the grain proportions measured by atomic force microscopy (AFM) and the unstressed grain bulks are omitted to magnify GB regions.

GBs emerging at higher heights. As an example, whereas the zipping of the right-hand GB in Fig. 1(a) (the shallowest) implies the generation of shear strain just within the bulk $B+C$, the zipping of the left-hand GB involves the whole drawn bulk $(A+B+C)$. It means that $\gamma_{e}$ increases (and so $\gamma$ decreases) as the section of the gap to close is deeper, and the involved volume is larger. This effect would give rise to anisotropies in the $\sigma_{N}$ strength along grain-junction contours with nohomogenous depths, $\sigma_{N}$ being higher where the gap is shallower [surface normal arrows in Fig. 1(a)].

Dynamics of stress accommodation: Evidences (I) and (III).- The stress accommodation for different degrees of $\sigma_{N}$ anisotropy is investigated by finite-element modeling (FEM) in two dimensions applied on a simplified configuration of three touching (in pairs) semispherical grains as shown in Fig. 1(a) (labeled $A, B$, and $C$ ). Such grains are not evenly spaced with each other, such that a deep gap appears between $A$ and $B$, and a shallow one between $B$ and $C$. The results are extrapolated to the model using periodic boundary conditions. The grains are elastically deformed by applying dissimilar normal loads $\sigma_{N}^{\text {left }}$ and $\sigma_{N}^{\text {right }}$ on their surface sections within the gaps $A-B$ and $B-C$, respectively. The $\sigma_{N}$ anisotropy is defined 
$\chi=\max \left(\sigma_{N}^{\text {right }}\right) / \max \left(\sigma_{N}^{\text {left }}\right) \geq 1$ for $\sigma_{N}^{\text {right }} \leq 100 \mathrm{MPa}$ taken within the typical range reported for ductile metal films [20] with $\max \left(\sigma_{N}\right)$ being reached close to grain junctions where $-\hat{\nabla}_{s}$ is parallel to $\gamma$.

Figures 1(b)-1(d) show the elastic deformation profiles (equilibrium profiles, hereafter) that result from different $\chi$ to compare them with the initial profile (solid curve). Full sequences of the pre-equilibrium evolutions are included in Ref. [18]. The evolution of grains subjected to isotropic tensile stress $(\chi \approx 1)$, which implies coalescence driven by pure radial interactions without shear strain, gives rise to an array of uniform gaps with the same depth [Fig. 1(b)]. This is because the $\sigma_{N}$ strength to induce GB zipping increases as the gap is deeper (i.e., $\sigma_{N} \propto \sin ^{2}[\arctan (m)]$; thus, the zipping between $A$ and $B$ prevails over that between $B$ and $C$, until both gaps have the same depth. Since the morphology for $\chi \approx 1$ in Fig. 1(b) does not agree with those formed by irregular multigrain structures [evidence (III)], we can rule out the radial interactions between grains (such as the traction forces and Laplace pressures) as being mainly responsible for the inside bundling-outside grooving phenomena. As $\chi$ rises, a bundling of the grains $B$ and $C$ occurs, giving rise to a multigrain structure, together with processes of unzipping for $1<\chi<10$ [Fig. 1(c)] and grooving for $\chi \geq 10$ [Fig. 1(d)] of the external GBs between structures (here $A-B$ ). A detailed inspection of the spatial distribution of the stress field inside the structures [enclosed regions in Figs. 1(b)-1(d)] reveals its dipolar geometry as depicted Fig. 1(e). Whereas the surface continues being under traction, the zipped region comes under compression, whose strength increases as the anisotropy rises up to reach a saturation for $\chi>20$. A dipolar field of the growth stress was proposed previously (although with a different origin) by Tello et al. [6] to address the insertion of leading adatoms by the gaps under traction into the GBs under compression. Since the macroscopic growth stress $\sigma_{\Sigma}$ (that measured through the Stoney equation) corresponds in a rough approximation to the area under the curves plotted in Fig. 1(e) (i.e., $\sigma_{\Sigma} \propto \int_{\text {depth }}\langle\sigma\rangle$, where $\langle\ldots\rangle$ denotes the longitudinal average), this can be divided into two contributions: $\int_{\text {shallow }}\langle\sigma\rangle+\int_{\text {deep }}\langle\sigma\rangle$, namely, shallow tensile stress + deep compressive stress, respectively. As displayed in the inset of Fig. 1(e), the deep compressive stress becomes the predominant contribution for $\chi>10$, which accounts for the compressive nature of $\sigma_{\Sigma}$ during the postcoalescence [evidence (I)], and it allows us to correlate the stress behavior with the observed phenomena of grain bundling and grooving [evidence (III)].

Kinetics of stress relaxation: Evidence (II).-Unlike the generation of the equilibrium profiles in Fig. 1 that happens at atomic vibration time scale $(\sim 0.1 \mathrm{ps})$, the relaxation of such profiles by atomic currents takes place at longer times $t$ within the range of surface $(\sim \mu \mathrm{s}-\mathrm{ms})$ and GB $(\sim \mathrm{sec}-\mathrm{h})$ diffusion time scales. Consequently, the kinetics of such a relaxation is approached by solving the following coupled mesoscopic rate equations that describe the evolution of the film surface $\left[S\left(\vec{r}_{R \phi}, t\right) \equiv S\right.$, by dynamic scaling in Eq. (2a)] and film density $\rho\left(\vec{r}_{R \phi z}, t\right) \equiv \rho(\vec{r}, t)$, Eq. (2b)] in-plane $(\hat{R}, \hat{\phi})$ and out-of-plane $(\hat{z})$ directions, respectively (details in Ref. [18]),

$$
\begin{aligned}
\partial_{t} S= & \int_{-\varphi / 2}^{\varphi / 2} F(\Theta) \partial \Theta-\kappa_{s} \nabla_{S}^{2}\left(\Omega\left[\gamma_{s}\left\{\nabla^{2} S+(\nabla S)^{2}\right\}-\sigma(S)\right]\right) \\
& +\eta\left(\vec{r}_{R \phi}, t\right), \\
\partial_{t} \rho= & -\kappa_{g b} \nabla_{\hat{z}}^{2}\left[\rho-\rho_{0}(\vec{r}, t)\right],
\end{aligned}
$$

where $F$ is the deposition flux, which (in general) impinges inhomogeneously on the surface given its dependence on the incidence angle $\Theta$ and the local aperture angle $\varphi\left(\vec{r}_{R \phi}\right)$-both measured from $-\hat{z}, \kappa_{s}$ and $\kappa_{g b}$ corresponds to the surface and GB diffusion coefficient, respectively, $\Omega$ is the atomic volume, and $\eta\left(\vec{r}_{R \phi}, t\right)$ denotes the uncorrelated noise to which both the deposition and diffusive noise contribute. The angular distribution of the flux $F(\Theta)$ depends on the flux-source characteristics while the aperture angles are determined by phenomena of shadowing and steering. Whereas the first term in Eq. (2a) considers the local advance of the surface due to the flux, the second one accounts for the lateral relaxation by stress-biased surface diffusion via surface currents $J_{s}=-\kappa_{s} \nabla_{s} \mu$ driven by the chemical potential $\mu \approx \Omega\left[\gamma_{s}\left(\nabla^{2} S+(\nabla S)^{2}\right)-\sigma(S)\right]$ resulting from the competition between the surface curvature $\propto \nabla^{2} S$, the slope $\propto(\nabla S)^{2}$ and the surface stress $\sigma(S)$. $J_{s}$ flows toward sites with higher curvatures and slopes (which implies higher densities of dangling bonds and steps, respectively) and/or under traction (those with lower film densities) to release the shallow tensile stress. Equation (2b) approaches the deep-compressive-stress relaxation (in terms of density evolution) through GB diffusions occurring preferentially toward the film surface (to where the density gradient is higher). Equations (2a) and (2b) are coupled to each other via the relationships: (density-surface) $\rho_{0}(\vec{r}, t)=\rho_{0} /\left\{1+e^{4\left[r_{z}-S\left(\vec{r}_{R \phi}, t\right)\right] / \omega}\right\}$, describing the density of the film-surface-vacuum system as a continuous sigmoid function varying within the surface region of width $\omega$ (surface roughness) between the unstrained film density $\rho_{0}$ and the null vacuum density, and (stress-density) $\sigma(\vec{r}, t) \propto-\nabla_{r} U\left[\rho(\vec{r}, t)^{-1 / 3}\right]$ connecting the stress with the film density through an embeddedatom potential $U$. For moderate stresses giving rise to small perturbations in the film density, $\sigma$ is estimated here as $\sigma(\vec{r}, t) \approx M\left[\sqrt[3]{\rho_{0}(\vec{r}, t) / \rho(\vec{r}, t)}-1\right]$ with $M$ being the elastic modulus.

Equations 2(a) and 2(b) are computed in 1 + 1D [i.e., in the plane $(\hat{R}, \hat{z})$ assuming radial symmetry] by the RungeKutta method. The integration is carried out within the enclosed regions in Figs. 1(c) and 1(d) using the equilibrium profiles by FEM as initial conditions, and assuming periodic boundary conditions. The physical constants for 
the calculation are summarized in Ref. [21]. Figure 2(a) displays the equilibrium profile by FEM for $\chi=4$ (that corresponds to $\sigma_{\Sigma}>0$ ), whose evolution driven by both surface and GB diffusion is outlined in Figs. 2(b) and 2(c), respectively, and expanded in Ref. [18]. The relaxation of the dipolar growth-stress field is plotted in Fig. 2(d). The fact that the surface diffusion differs in several orders of magnitude from that of GB $\left(\kappa_{s} / \kappa_{g b} \sim 10^{12}[22,23]\right)$ allows us to separate their contributions to the stress relaxation by time scales. This means that during the relaxation of $\int_{\text {shallow }}\langle\sigma\rangle$ by surface diffusion happening at tens of $\mathrm{ms}$ [Fig. 2(d)], the GB diffusion is practically frozen and $\int_{\text {deep }}\langle\sigma\rangle$ remains constant $( \pm 10 \%)$ for few seconds. The delay in the release of $\int_{\text {deep }}\langle\sigma\rangle$ is also increased by the fact that the surface diffusion moves the surface away from the compression core. Consequently, the $\int_{\text {deep }}\langle\sigma\rangle$ contribution to $\sigma_{\Sigma}$ becomes higher (note the system turns out to be under compression for $t>10 \mathrm{~ms}$ ).

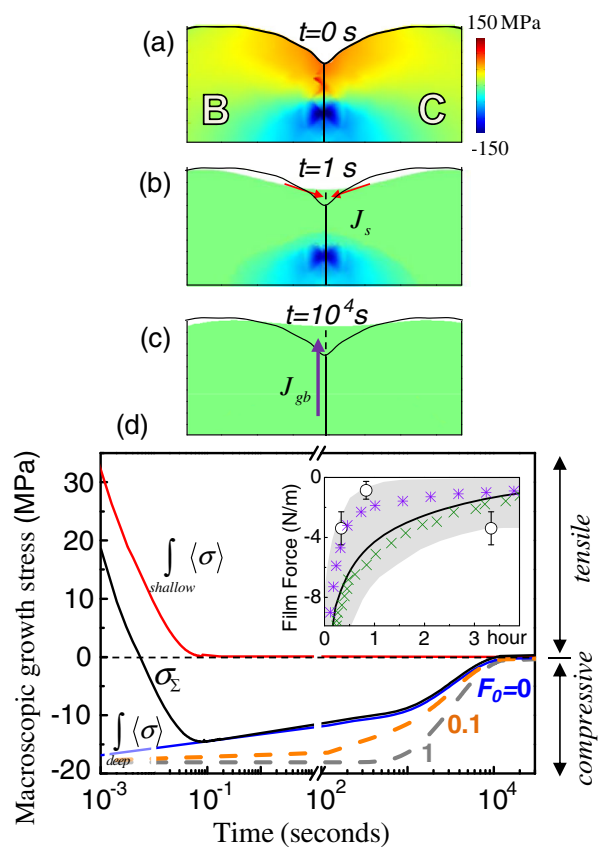

FIG. 2 (color online). (a)-(c) Relaxation of the growth stress generated by interactions between the grains $B$ and $C$ with $\chi=4$ by surface (b) and GB (c) diffusions for different relaxation times once the deposition flux is stopped $\left(F_{0}=0\right)$. The color scale denotes the strength of the stress field whereas the solid lines depict the outline of the equilibrium profile by FEM. (d) Evolution of $\int_{\text {shallow }}\langle\sigma\rangle$ and $\int_{\text {deep }}\langle\sigma\rangle$ contributions to $\sigma_{\Sigma}$ for different $F_{0}$ as labeled. Inset: fit of the simulated $\sigma_{\Sigma}$ relaxation (expressed as film force $\sigma_{\Sigma} \lambda_{\perp}$ with $\lambda_{\perp}=105 \mathrm{~nm}$ [13] by Scherrer) to the residual stress extrapolated from data reported for $\mathrm{Au}$ films measured in situ by capacitance ( $\times$ [24]) and cantilever beam methods (* [25]) and ex situ by grazingincidence DRX (open circles [13]). Dashed region corresponds to the data dispersion.
The slow relaxation of the deep compressive stress (plotted in terms of film force $\sigma_{\Sigma} \lambda_{\perp}$ with the out-of-plane grain size $\lambda_{\perp}$ estimated in Ref. [13] from the Scherrer's equation) is confirmed in the inset of Fig. 2(d) by fitting our model to experimental data reported for polycrystalline $\mathrm{Au}$ films, whose residual stresses were measured at RT by complementary techniques: (a) Through the Stoney equation determining in situ the system curvature by capacitance [24] and (b) using the standard $\sin ^{2} \psi$ method from ex situ measurements by grazing-incidence XRD [13]. $\kappa_{g b}=5 \times 10^{-15} \mathrm{~cm}^{2} / \mathrm{s}$ resulting from the fit shows a good agreement with those previously reported [23], which supports our hypothesis of deep-compression release through GB diffusion. Nevertheless, other faster relaxations $(\sim$ few min) are also viable; e.g., Leib et al. $[9,25]$ proposed a temperature-independent relaxation based on obstacle-free dislocation glides for $\mathrm{Au}$ films on $\mathrm{SiN}_{x} / \mathrm{Si}(100)$ [symbol $*$ in the inset of Fig. 2(d)].

In this scenario, the deposition flux $F$ influences the stress behavior in two manners. (a) It modifies the equilibrium profile as a consequence of its nonuniform nature, and/or (b) limits partially (or totally) the mass transport-driven relaxations happening at deposition time scales $\geq 1 / F_{0} \lambda^{2}\left(\geq 1 / F_{0}\right)$. The first manner (a) would take place, for example, during growths under lowmobility conditions in which the surface diffusion is not enough to compensate higher growth rates on grain tops induced by shadowing and steering. Thus, the gaps become deeper, the grain sections under traction are larger, and the tensile stress prevails. This effect, which is counterbalanced by the surface diffusion when the flux is stopped and vice versa, would give rise to similar stress behavior to that reported by Koch et al. [5] for Fe films deposited at low temperatures $(T=310 \mathrm{~K})$. Conversely (i.e., under high mobility conditions), the shallower gaps (those with higher aperture angles) receive higher fluxes than the deeper ones that contribute to speeding up the filling rate of the former and, consequently, increasing the $\sigma_{N}$ anisotropy that causes the deep compressive stress to strengthen [Fig. 1(e)]. The second manner $(b)$ occurs when the flux hinders the release of $\int_{\text {deep }}\langle\sigma\rangle$ (Fig. 2(d) - curves for different $F_{0}$ ) by moving the surface away from the compression core faster than the mass transport through GB diffusion, which is the responsible for the stress relaxation during periods of flux interruption and postdeposition $\left(F_{0}=0\right)$. Note that the fact that realistic fluxes $\left(F_{0} \ll 10 \mathrm{~nm} / \mathrm{s}\right)$ cannot limit the shallow relaxation by surface diffusion under high mobility conditions, it reduces the $\sigma_{N}$ anisotropy threshold required to induce a macroscopic compressive stress down to practical values (e.g., $\chi \geq 1.5$ for $F_{0}=0.1 \mathrm{~nm} / \mathrm{s}$ after $t=1 \mathrm{~s}$ not shown) lower than the threshold of $\chi \geq 10$ by FEM for kinetically frozen systems [inset of Fig. 1(e)]. Different combinations of the effects described above account for the reversible 
dependence on the flux of the postcoalescence growth stress [evidence (II)].

In conclusion, we provide major findings on (i) the key role played by interactions between grains (in particular those azimuthally driven by the misorientation between coalescing grains) at complementary space scales on the origin, nature, and dipolar structure of the postcoalescence growth stress; and (ii) how the interplay between the deposition conditions (i.e., flux and mobility) and the mechanisms of stress accommodation-relaxation determines the growth stress behavior in regard to its reversible dependence on the flux and evolution at different time scales. The fact that the simulated results allow a successful interpretation of the main experimental evidences reported so far supports the soundness of the proposed inside bundling-outside grooving model.

Work supported by the projects 200960 I182 (CSIC), CCG10-UAM/MAT-5537 (Comunidad de Madrid and Universidad Autónoma de Madrid), and AYA2010-22032. A. G.-G. acknowledges the financial support of CSIC under Project No. 201160E053.

*Corresponding author. agonzo@icmm.csic.es

[1] A. Witvrouw, H. A.C. Tilmans, and I. De Wolf, Microelectron. Eng. 76, 245 (2004).

[2] S. Kim, J. Electrochem. Soc. 158, H904 (2011).

[3] J.-H. Cho, T. James, and D. H. Gracias, Adv. Mater. 22, 2320 (2010).

[4] L. B. Freund and S. Suresh, Thin Film Materials: Stress, Defect Formation, and Surface Evolution (Cambridge University Press, Cambridge, England, 2003).

[5] R. Koch, D. Hu, and A. K. Das, Phys. Rev. Lett. 94, 146101 (2005).

[6] J. S. Tello, A. Bower, E. Chason, and B. Sheldon, Phys. Rev. Lett. 98, 216104 (2007) and references therein.

[7] J. W. Shin and E. Chason, Phys. Rev. Lett. 103, 056102 (2009) and references therein.

[8] C. Friesen and C. V. Thompson, Phys. Rev. Lett. 93, 056104 (2004).

[9] J. Leib and C. V. Thompson, Phys. Rev. B 82, 121402(R) (2010).

[10] C.-W. Pao, S. Foiles, E. Webb, D. Srolovitz, and J. Floro, Phys. Rev. Lett. 99, 036102 (2007).
[11] B. Fu, W. An, C. Turner, and G. Thompson, Phys. Rev. Lett. 105, 096101 (2010).

[12] E. Chason, J. W. Shin, S. J. Hearne, and L. B. Freund, J. Appl. Phys. 111, 083520 (2012).

[13] A. González-González, G. M. Alonzo-Medina, A. I. Oliva, C. Polop, J. L. Sacedón, and E. Vasco, Phys. Rev. B 84, 155450 (2011).

[14] A. A. Navid, E. Chason, and A. M. Hodge, Surf. Coat. Technol. 205, 2355 (2010).

[15] Stage denoted as "island coalescence regime" in C. Polop, C. Rosiepen, S. Bleikamp, R. Drese, J. Mayer, A. Dimyati, and T. Michely, New J. Phys. 9, 74 (2007).

[16] W. Zhang, F. Ma, T. Zhang, and K. Xu, Thin Solid Films 520, 708 (2011).

[17] J. W. Shin and E. Chason, J. Mater. Res. 24, 1522 (2009).

[18] See Supplemental Material at http://link.aps.org/ supplemental/10.1103/PhysRevLett.110.056101 for details concerning Eqs. (1) and (2), and movies showing both the evolution of the growth stress for different $\chi$, and its relaxation by surface and bulk diffusions for $\chi=4$.

[19] D. Moldovan, D. Wolf, and S. R. Phillpot, Acta Mater. 49, 3521 (2001).

[20] Maximum values of tensile stress measured in (Ag) $\sigma_{N} \leq$ $190 \mathrm{MPa},(\mathrm{Al}) \leq 120 \mathrm{MPa}$, and $(\mathrm{Au}) \leq 225 \mathrm{MPa}$; from J. A. Floro, S. J. Hearne, J.A. Hunter, P. Kotula, E. Chason, S. C. Seel, and C. V. Thompson, J. Appl. Phys. 89, 4886 (2001), and Ref. [13].

[21] Elastic constants: (Young's modulus) $E=14.4 \mathrm{GPa}$, (Poisson ratio) $v=0.44$, (elastic modulus for polycrystalline films) $M=E /(1-v)$. Structural parameters: (atomic volume) $\Omega=12.5 \times 10^{-3} \mathrm{~nm}^{3}$. Thermodynamic and kinetics constants: (surface energy) $\gamma_{s}=1.54 \mathrm{~J} / \mathrm{m}^{2}$ [13], (surface diffusion coefficient) $\kappa_{s}=1 \times 10^{-2} \mathrm{~cm}^{2} / \mathrm{s} \quad$ [22], (GB diffusion coefficient) $\kappa_{g b}=4.2 \times 10^{-15} \mathrm{~cm}^{2} / \mathrm{s} \quad$ [23]. Growth parameters [13]: (flux) $F(\Theta) \approx F_{0}$-plane flux with $F_{0}=0.1-1.0 \mathrm{~nm} / \mathrm{s}$, (uncorrelated noise) $\eta=0$, (film roughness) $\omega=1.8 \mathrm{~nm}$, (grain size) $\lambda>\lambda_{0} \approx 140 \mathrm{~nm}-$ threshold for shear-stress generation, and (deposition temperature) $T=373 \mathrm{~K}$.

[22] C. L. Liu, J. M. Cohen, J. B. Adams, and A. F. Voter, Surf. Sci. 253, 334 (1991).

[23] D. Gupta, J. Appl. Phys. 44, 4455 (1973); Phys. Rev. B 7, 586 (1973).

[24] R. Abermann and R. Koch, Thin Solid Films 129, 71 (1985).

[25] J. Leib, R. Mönig, and C. V. Thompson, Phys. Rev. Lett. 102, 256101 (2009). 